et enseignement

\title{
L'adaptation du modèle de la transposition didactique à l'éducation préscolaire : un éclairage nouveau sur le rôle de l'enseignante lors du jeu symbolique pour faire émerger l'utilisation des savoirs mathématiques chez les enfants
}

\author{
Isabelle Deshaies ${ }^{1}$ et Manon Boily ${ }^{2}$ \\ ${ }^{1}$ Université du Québec à Trois-Rivières, Québec, Canada \\ ${ }^{2}$ Université du Québec à Montréal, Québec, Canada
}

\begin{abstract}
Pour citer cet article :
Deshaies, I. et Boily, M. (2021). L'adaptation du modèle de la transposition didactique à l'éducation préscolaire : un éclairage nouveau sur le rôle de l'enseignante lors du jeu symbolique pour faire émerger l'utilisation des savoirs mathématiques chez les enfants. Didactique, 2(2), 84-114. https://doi.org/10.37571/2021.0205.
\end{abstract}

Résumé : La présente recherche porte sur la mise en place de l'adaptation du modèle de transposition didactique de Chevallard (1991) à l'éducation préscolaire. Cette recherche qualitative réalisée auprès de deux enseignantes et 33 enfants d'âge préscolaire vise deux objectifs : 1) identifier la posture de l'enseignante quant à son implication dans le jeu symbolique des enfants avant et après la mise en place d'un dispositif de formation;2) décrire les rôles adoptés par l'enseignante dans le jeu symbolique des enfants, à la suite d'un dispositif de formation, en lien avec l'opérationnalisation d'une transposition didactique interne des savoirs éveillés vers les savoirs utilisés en mathématiques. Une analyse de contenu thématique déductive (Braun et Clarke, 2006) a été réalisée à partir du cadre théorique de Lemay et al., 2017, adapté de Johnston et al., 2005 qui ont identifié quatre rôles que l'enseignante peut adopter dans le jeu des enfants. Les analyses mettent en évidence un changement de posture chez les enseignantes ainsi que l'adoption de trois nouveaux rôles dans les jeux des enfants : observateur, cojoueur et 
leader de jeu. Les résultats permettent de porter un regard nouveau sur l'importance du rôle occupé par les enseignantes lors du jeu symbolique afin de permettre à l'enfant d'utiliser ses savoirs mathématiques et ce, en respectant les fondements de l'approche développementale.

Mots-clés : éducation préscolaire ; transposition didactique ; savoirs mathématiques; approche développementale ; jeu symbolique ; rôle de l'enseignante 


\section{Problématique}

L'une des visées du gouvernement concernant l'éducation préscolaire est de proposer un environnement sécurisant et bienveillant afin d'offrir des chances égales à tous les enfants de 4 et 5 ans de se développer selon leur plein potentiel (Ministère de l'Éducation et de l'Enseignement supérieur [MEES], 2017, 2018). Le programme-cycle à l'éducation préscolaire a pour objectif de favoriser le développement global de tous les enfants et spécifie que l'enfant :

Acquiert alors des habiletés et fait des apprentissages se situant dans sa zone proximale de développement, c'est-à-dire entre son niveau de développement actuel (ce qu'il est capable de faire seul) et son niveau de développement potentiel (ce qu'il est capable d'accomplir avec l'aide de pairs plus habiles que lui ou d'un adulte). (Ministère de l'Éducation du Québec [MEQ], 2021, p.4).

En outre, l'enseignante a un rôle important à jouer notamment en initiant l'enfant à des « connaissances (savoirs) » (MEQ, 2021, p.14) intégrées au domaine d'apprentissage de la mathématique afin qu'il puisse élaborer sa pensée et construire sa compréhension du monde.

\section{Les premiers apprentissages en mathématiques}

Plusieurs recherches en didactique des mathématiques montrent que les premiers apprentissages en mathématiques jouent un rôle dans le fait d'éprouver ou non des difficultés dans cette discipline, et aussi que les habiletés précoces en mathématiques sont un important prédicteur de la réussite scolaire (Clark et al., 2010 ; García Coll et al., 2007 ; Rourke et Conway, 1997). Les notions mathématiques en jeu sont souvent celles qui devraient être acquises durant la période préscolaire ou dès les premières années de scolarisation (Garcia Coll et al., 2007) et font référence, entre autres, aux savoirs liés au dénombrement (Noël, 2005).

\section{L'enseignante à l'éducation préscolaire}

Actuellement, la formation initiale des enseignantes offre seulement deux ou trois cours concernant l'éducation préscolaire. Il est donc possible que les enseignantes ne disposent pas de l'ensemble des notions mathématiques à enseigner au cycle préscolaire et que cela suscite chez elles un faible sentiment d'efficacité professionnelle. D'ailleurs, comme le mentionne l'étude de Casavant et Nunez-Moscoso (2020), qui porte sur l'insertion professionnelle, les enseignantes reconnaissent qu'il est essentiel de se sentir compétents dans la ou les matières qu'elles enseignent. Or, qu'elles exercent au préscolaire ou au 
primaire, elles se disent souvent compétentes pour enseigner le français, mais ne mentionnent pas les mathématiques, ce qui laisse entendre une lacune dans les connaissances qu'elles ont dans cette discipline fondamentale (Casavant et NunezMoscoso, 2020). Le programme de l'éducation préscolaire laissant une large place à l'autonomie professionnelle des enseignantes, les enfants arrivent en première année avec des connaissances et des compétences variées, malgré toute la stimulation et les efforts des enseignantes du préscolaire. En effet, une étude de Pagani et ses collègues (2010) démontre qu'une certaine proportion d'enfants ne franchit pas la maternelle munie des acquis nécessaires pour réussir en arithmétique dès la première année.

\section{Les différents types d'approches présents à l'éducation préscolaire}

À l'éducation préscolaire, différentes approches sont présentes pour soutenir les apprentissages des enfants, mais plus particulièrement deux ; l'approche développementale et l'approche scolarisante. L'approche développementale favorise le développement global de tous les enfants (MEQ, 2021) et s'inscrit dans une perspective d'apprendre pour jouer (Marinova, 2014). Dans cette approche, l'enfant bénéficie de toute la latitude pour diriger son activité et réaliser ses apprentissages. Afin d'optimiser ce type d'activité, l'intervention de l'adulte, sous forme de questionnement et d'étayage, est essentielle (Boily et Deshaies, 2021). L'approche scolarisante vise davantage l'apprentissage de contenus liés aux matières scolaires en proposant des activités d'apprentissage plus formelles (Organisation de Coopération et de Développement économique [OCDE], 2007; Boily et Deshaies, 2021).

Puisque le programme cycle à l'éducation préscolaire mentionne que chaque enfant puisse « bénéficier chaque jour de deux périodes de 45 à 60 minutes de jeu libre où il choisira avec qui et avec quoi il jouera »(MEQ, 2021, p.9), la présence de ces deux approches apporte une certaine confusion au sein des milieux et suscite un questionnement quant à la posture à adopter lors des jeux libres de l'enfant (Conseil Supérieur de l'Éducation [CSE], 2012). Comment soutenir la compréhension de l'enseignante vis-à-vis le rôle à jouer lors du jeu libre ou jeu symbolique, afin de favoriser l'utilisation des savoirs mathématiques de l'enfant tout en respectant l'approche développementale? 


\section{Cadre conceptuel}

\section{Le modèle de la transposition didactique}

Pour comprendre le mécanisme d'utilisation des rôles de soutien et d'accompagnement de l'enseignante (Johnston et al., 2005), en vue de permettre à l'enfant l'utilisation de savoirs mathématiques lors du jeu symbolique, nous prenons appui sur le modèle de transposition didactique de Chevallard (1991). Nous pouvons définir ce dernier comme étant : "Un contenu de savoir ayant été désigné comme savoir subi [...] un ensemble de transformations adaptatives qui vont le rendre apte à prendre sa place parmi les objets d'enseignement. Le " travail " qui d'un objet de savoir à enseigner fait un objet d'enseignement est appelé la transposition didactique » (Chevallard 1991, p.39). En fait, le modèle de transposition didactique tel que conçu par Chevallard (1991), fait subir aux savoirs savants une série de transformations les rendant aptes à devenir des objets d'enseignement. Deux types de transposition s'opèrent ; soit celle externe et celle interne. La transposition didactique externe « représente le processus de transformation, d'interprétation et de ré-élaboration didactique du savoir scientifique constitué dans différents domaines de connaissances » (Paun, 2006, p.4). Lors de cette étape du processus de transposition, s'articulent le savoir savant, qui est celui validé et labellisé comme étant le savoir scientifiquement correct par les chercheurs et le savoir à enseigner qui est celui défini par les programmes et sur lequel les professionnels de l'enseignement s'appuient. Paun (2006) appelle cette transposition le curriculum formel ou prescrit ; puisqu'il suppose une transformation entre les savoirs savants qui doivent se traduire en savoirs à enseigner. La transposition didactique interne " représente l'ensemble des transformations successives et négociées subies par le curriculum formel dans le cadre de processus d'enseignement et d'apprentissage, tout au long du parcours professeur-élève » (Paun, 2006, p.8). Comme le précise Paun (2006, p.8), cette transposition se nomme interne puisqu'elle « se produit à l'intérieur de la relation professeur-élève. » Dans ce modèle, Paun (2006) fait référence à deux types de curriculums; soit le curriculum réel et le curriculum réalisé. Le curriculum réel fait référence au savoir enseigné tel que défini par Chevallard (1991) et « représente le résultat des transformations subies par le curriculum formel, dans son parcours de professeur à l'élève et à l'intérieur du processus d'enseignement » (Paun, 2006, p.8).

Le curriculum réalisé fait référence au savoir appris et retenu tel que défini par Chevallard (1991). Comme le précise Paun (2006, p.8), il « est constitué d'un ensemble d'expériences éducatives négociées $[\ldots]$ exprimant le rapport particulier de l'élève au savoir scolaire. » Ainsi, le processus de transposition didactique ne s'inscrit pas dans un processus de 
transmission de curriculum par l'enseignant, mais bien par une négociation entre le curriculum réel et le curriculum réalisé qui représente ultimement le savoir appris ou retenu chez l'élève.

Dans un cadre s'intéressant à l'éducation préscolaire où l'approche développementale est mise de l'avant (MEQ, 2021), certains ajustements doivent être apportés au modèle de transposition didactique proposé par Chevallard (1991). D'abord, puisque le mandat de l'éducation préscolaire est de favoriser le développement global de tous les enfants et plus spécifiquement, en mettant les bases de la scolarisation (MEQ, 2021), le programme fait référence à l'éveil des connaissances (savoirs) chez l'enfant et non l'enseignement spécifique de connaissances (savoirs). De plus, comme le mentionnent Clements et Sarama (2012) ainsi que Starkey et Klein (2000), une importance particulière doit être mise sur les activités d'éveil en mathématiques à la petite enfance et non sur un enseignement formel des savoirs. Ainsi, dans la proposition de notre modèle, nous substituons le terme « savoir à enseigner » par « savoir à éveiller » et le terme « savoir enseigné » par " savoir éveillé ». Précisons que ce «savoir éveillé » est possible grâce à la mise en place de situations pédagogiques ludiques de la part de l'enseignant. Ainsi, le processus de transposition didactique demeure le même, seules les terminologies et le soutien offert par l'enseignant diffère pour mieux cadrer dans le programme cycle à l'éducation préscolaire (MEQ, 2021). Nous substituons également le terme « savoir appris » par le terme " savoir utilisé lors des jeux symboliques ». Comme le souligne le programme cycle à l'éducation préscolaire (MEQ, 2021, p. 7) ; « soutenu par des interventions, l'enfant s'engage dans des situations d'apprentissage issues du monde du jeu et de ses expériences de vie et commence à jouer son rôle d'élève actif. » Ainsi, l'enfant est amené à utiliser ses savoirs éveillés lors des situations pédagogiques ludiques initiées par l'enseignante dans ses périodes de jeu symbolique. De surcroit, les termes substitués du modèle de transposition didactique de Chevallard (1991) n'entravent pas le rôle de l'enseignant lors de la transposition didactique externe puis interne, mais permettent l'adaptation aux spécificités de l'éducation préscolaire. Ainsi, le respect du rythme, des besoins et des intérêts de l'enfant sont présents et il y a priorisation des activités initiées par l'enfant (Marinova et Drainville, 2019) ; ce qui est en accord avec le programme-cycle (MEQ, 2021).

\section{L’approche développementale}

L'approche développementale a comme visée la préparation de l'enfant à sa vie plutôt qu'à l'école (OCDE, 2007). Il s'agit d'une approche holistique où tous les aspects du développement de l'enfant sont abordés afin de favoriser son développement global 
(OCDE, 2007). Dans cette approche, l'enfant a une place centrale puisqu'il est le maître d'œuvre des apprentissages qu'il réalise (OCDE, 2007). Au sein de celle-ci, l'adulte ayant un rôle de soutien aux apprentissages est invité à proposer des activités d'éveil à l'enfant en tenant compte de ses intérêts (OCDE, 2007). Le but étant que l'enfant apprenne dans des contextes et des situations qui ont du sens à ses yeux (OCDE, 2007). Drainville (2017) parle d'aborder les apprentissages des enfants « dans un contexte authentique, soit lors de situations réelles tirées du vécu des enfants»(p.26). Le programme à l'éducation préscolaire va dans ce sens puisqu'il suggère l'offre d'un environnement où le jeu est priorisé et le soutien de l'adulte dans les apprentissages des enfants est valorisé : «Un environnement riche où le jeu, notamment le jeu symbolique, est amorcé par l'enfant et soutenu par l'adulte permet à l'enfant d'explorer, de créer, d'improviser, de jouer un rôle, de manipuler, etc. » (MEQ, 2021, p.9). Dans le contexte de cette étude, l'apprentissage des mathématiques s'inscrivant dans une approche développementale respectera les conditions suivantes : le jeu comme moyen d'apprentissage, le soutien de l'adulte aux apprentissages de l'enfant, l'offre d'un environnement riche tiré de situations de la vie, l'intérêt de l'enfant et le respect du rythme de ses apprentissages. Les situations de la vie dans le cadre de cette étude, sont celles qui émergent des jeux de l'enfant lorsqu'il est dans les centres d'apprentissage (jeu symbolique).

\section{La place du jeu dans le développement de l'enfant au préscolaire et le rôle que l'adulte peut $y$ tenir}

À l'éducation préscolaire, les enseignantes sont invitées à proposer à l'enfant des activités et à offrir des environnements d'apprentissage qui mettent en valeur le jeu puisque celuici constitue «sa manière privilégiée d'apprendre » (MEQ, 2021, p.9). À cet égard, le jeu symbolique est choisi en raison de ses vertus. Pour permettre à l'enfant de naviguer à travers cette forme de jeu, la construction de centres d'apprentissage tels que ceux où l'enfant pourra y tenir des rôles (médecin, pâtissier, fleuriste) est alors conseillé (Ministère de l'Éducation de l'Ontario, 2006). L'enfant peut donc initier ses activités de jeu alors qu'il est invité à faire des choix, à vivre ses propres découvertes et à créer ses propres scénarios (MEQ, 2021). Toutefois, l'adulte peut tenir une place importante dans le jeu de l'enfant, principalement celle où il a un rôle de soutien aux apprentissages de l'enfant afin de lui permettre d'approfondir « sa vision du monde pour s'approprier la réalité » (MEQ, 2021, p.9). Il s'agit alors d'adopter la perspective de Vygotski (1934, 2012) qui propose de donner à l'adulte un rôle dans les apprentissages de l'enfant afin de l'amener à réaliser ceux qui se trouvent dans sa zone proximale de développement. Le soutien de l'enseignante envers les enfants sert alors à «élargir leur répertoire d'actions, à approfondir leur 
compréhension de différents concepts et à enrichir leurs connaissances...» (MEQ, 2021, p.8). À cet égard, l'enseignante peut proposer plusieurs types d'activités prenant la forme de situations d'apprentissage à partir d'un jeu (ex. : elle propose un jeu lors duquel les enfants doivent trouver toutes les combinaisons possibles de réglettes Cuisenaire pour combler la remorque d'un camion selon le nombre inscrit), mais elle peut tout aussi bien s'insérer dans les situations d'apprentissage qui émergent du jeu (ex. : elle occupe un rôle de cojoueur dans le coin poupée et elle classe les vêtements des poupées; en l'observant, les enfants utilisent leur capacité de classement également).

\section{Le jeu de plus haut niveau : le jeu symbolique}

Le jeu de plus haut niveau, à l'éducation préscolaire, est celui du jeu symbolique (Marinova et Biron, 2010). Ce dernier constitue l'activité maitresse chez les enfants d'âge préscolaire pour réaliser des apprentissages de haut niveau (Marinova, 2015). Lors de ce type de jeu de l'enfant, des situations d'apprentissage peuvent émerger et l'enseignante peut les utiliser pour amener les enfants à transposer leurs savoirs éveillés lors des situations antérieures proposées par l'enseignante; il s'agit des situations d'apprentissage issues du jeu. Dans cette perspective, comme le mentionne Marinova (2015), ces situations sont :

Situées entre le jeu et les activités d'apprentissage de type scolaire, les situations d'apprentissage issues du jeu reflètent le principe de continuité des activités humaines, lequel est un principe de base de la théorie de l'activité de Leontiev. Étant un moment dans le jeu, elles témoignent de la naissance de l'activité d'apprentissage au sein de l'activité ludique. (p.110)

Par ailleurs, ces situations d'apprentissage issues du jeu exigent de l'enseignante qu'elle puisse s'adapter au monde imaginaire du jeune enfant (Marinova, 2015).

À l'éducation préscolaire, il est suggéré d'offrir aux enfants un environnement qui leur permet de «s'initier à de nouvelles connaissances liées aux domaines d'apprentissage » tel que la mathématique (MEQ, 2021, p.16). Pour ce faire, il est proposé que l'adulte puisse s'impliquer auprès des enfants par le questionnement, le dialogue et la rétroaction (MEQ, 2021). Dans la perspective du « jeu guidé », tel que défini par Toub et ses collègues (2016), la place que prend l'adulte augmente, pour l'enfant, la possibilité qu'il fasse de nouveaux apprentissages. Un des fondements de cette approche a trait à l'implication de l'adulte pour soutenir le développement de l'enfant et enrichir ses apprentissages, voire de faire émerger de nouvelles connaissances. Dans le contexte de notre étude, les enseignantes ont été invitées à participer aux situations d'apprentissage issues du jeu des enfants et à les utiliser 
pour amener ces derniers à transposer leurs savoirs éveillés lors de situations initiées par l'enseignante vécues au préalable.

\section{La situation pédagogique ludique initiée par l'enseignante versus la situation de jeu émergeant du jeu symbolique}

Plusieurs types de situations d'apprentissage peuvent être observées à l'éducation préscolaire. Notre étude en a identifié deux. La première situation, initiée par l'enseignante, se situe dans une perspective de jouer pour apprendre (Marinova, 2015); d'où son appellation de situation pédagogique ludique. Comme le mentionne Marinova (2015), le jeu devient «prétexte à l'apprentissage» (p.103). Lors de ce type de situation, «l'enseignante prend une posture didactique; elle conçoit, planifie et dirige le jeu comme toute autre activité d'apprentissage [...] » (Marinova, 2015, p.104). La deuxième situation, émergeant du jeu, se situe dans une perspective d'apprendre pour jouer; « le jeu est pensé et vécu comme un contexte d'apprentissage » (Marinova, 2015, p.104). Ainsi, pour jouer et faire évoluer le jeu, les enfants ont besoin de connaissances/savoirs. Lors de ce type de situation, comme le précise Marinova (2015), «l'enseignante prend une posture ludique : elle accompagne les enfants en jouant et en soutenant leur jeu [...]» (p.104). L'enseignante doit non seulement détenir les connaissances/savoirs à éveiller, proposer des situations pédagogiques ludiques permettant de les éveiller, mais également, par l'observation, déceler un scénario de jeu construit par les enfants qui lui permettrait de pousser l'utilisation de savoir plus loin pour amener les enfants à le réinvestir dans leur scénario de jeu. Ainsi, cet article fait état de la situation d'apprentissage où l'enseignante s'inscrit dans le jeu symbolique des enfants dans le but de le faire évoluer. Il rend compte de l'implication de l'enseignante dans les jeux des enfants pour favoriser chez celui-ci l'utilisation des savoirs éveillés précédemment lors des situations pédagogiques ludiques. La figure 1 résume notre modèle de transposition didactique à l'éducation préscolaire inspirée des travaux de Chevallard (1991). 


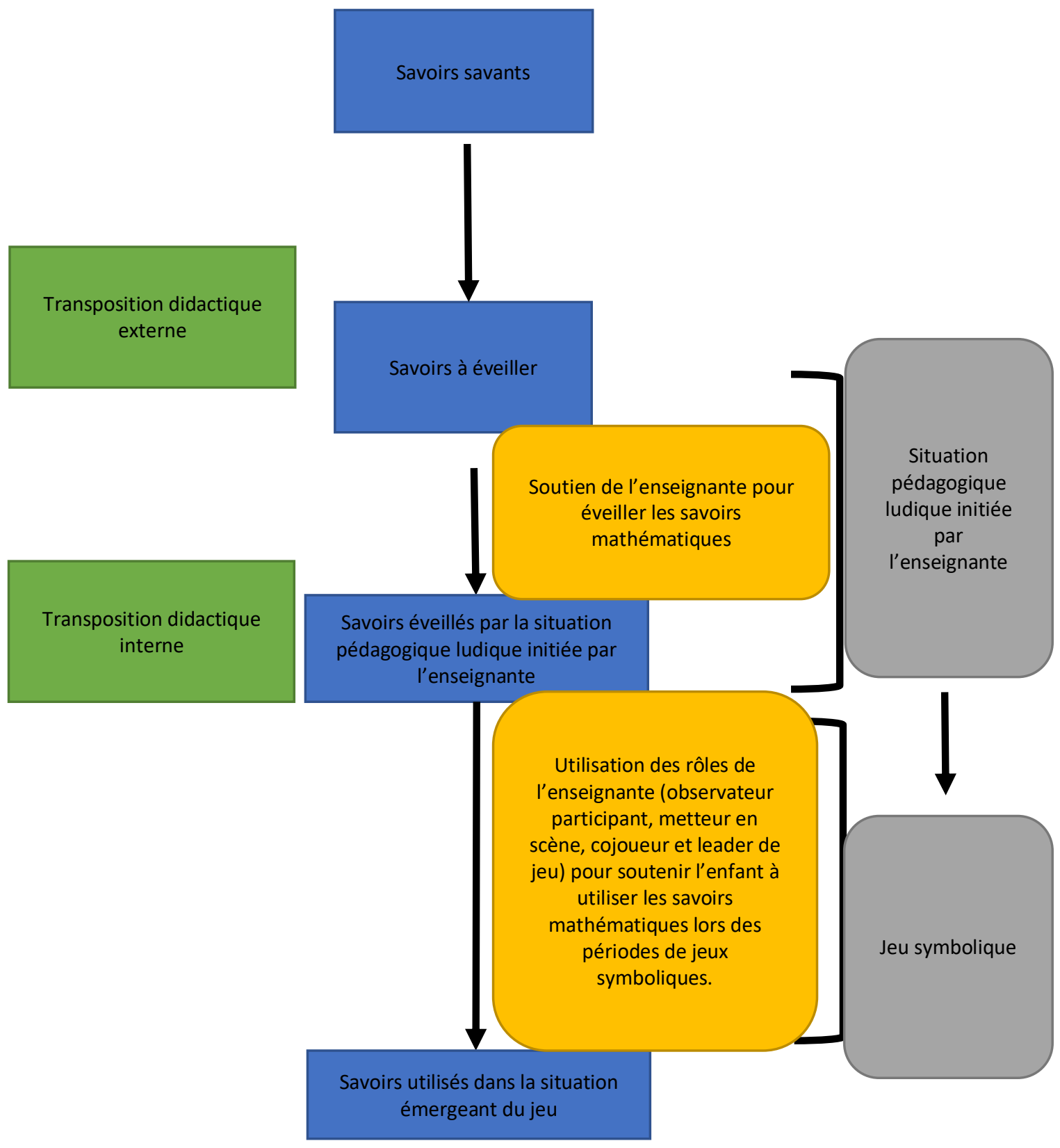

Figure 1. Modèle de transposition didactique à l'éducation préscolaire, schéma adapté de Chevallard (1991).

\section{Les différents rôles joués par les enseignantes lors des jeux symboliques}

Selon Johnson et al., (2005), certaines stratégies adoptées par les adultes permettraient d'accompagner le jeu des enfants, d'autres pourraient plutôt le limiter, lui nuire ou même 
interférer le cours de son déroulement. En ce sens, l'adulte peut jouer différents rôles à l'intérieur du jeu. Ces derniers se situant sur un continuum allant de l'absence d'accompagnement dans le jeu des enfants (désengagé), à un rôle d'observateur, de metteur en scène, de cojoueur, de leader de jeu, jusqu'à un accompagnement qui le domine (directeur/redirecteur de jeu) (Lemay et al., 2017, adapté de Johnston et al., 2005). Les quatre rôles qui se situent entre les extrémités seraient plus susceptibles d'encourager et de soutenir le jeu des enfants et seraient donc à privilégier lors du jeu symbolique (Lemay et al., 2017). Voici une description de chacun d'eux.

Lorsqu'elle occupe le rôle d'observateur, l'enseignante ne participe pas au jeu de façon active. Comme le mentionnent Point et Leclaire (2020) «dans ce rôle, l'adulte se positionne près des enfants qui jouent [...] il regarde les enfants et acquiesce ou fait d'autres signes non verbaux » (p.55). Lorsqu'elle occupe ce rôle, l'enseignante comprend ce que l'enfant reproduit dans son jeu symbolique, mais elle fait aussi comprendre à l'enfant que son jeu est important.

Lorsqu'elle occupe un rôle de metteur en scène, l'enseignante soutient les enfants qui organisent leur jeu et elle peut également les aider à aménager l'espace du coin jeu symbolique (Point et Leclaire, 2020). Lorsqu'elle occupe un rôle de cojoueur, l'enseignante participe directement au jeu des enfants. En fait, comme le précisent Point et Leclaire (2020), l'adulte et l'enfant participent de façon équitable dans le jeu. Ce rôle offre l'opportunité à l'adulte d'être un modèle pour l'enfant.

Enfin, lorsqu'elle occupe un rôle de leader de jeu, l'enseignante « devient un cojoueur dont les suggestions influencent significativement le scénario de jeu afin de l'enrichir et de le faire durer, en introduisant de nouveaux objets ou des éléments du scénario » (Point et Leclaire, 2020, p.57).

La présence de ces rôles soutiendrait l'émergence des savoirs lors du jeu symbolique de l'enfant (Lemay et al., 2017), dont ceux en mathématiques.

\section{Un regard sur l'émergence de l'arithmétique à l'éducation préscolaire}

Comme le mentionne le programme-cycle à l'éducation préscolaire (MEQ, 2021), « l'enfant entame à l'âge de 4 à 6 ans une transition d'un raisonnement perceptif vers un raisonnement logique qui lui offre un nouveau regard sur le monde » (p.48). Au regard des mathématiques, il est question ici de la transition entre le sens intuitif des nombres (sens 
des nombres) (Dehaene, 2011) et le lien entre ce sens des nombres et le nombre sous sa forme symbolique (Deshaies et al., 2015). Ce lien fait référence aux habiletés de dénombrement (Bideaud et al., 2004; Gelman, 1972; Gelman et Meck, 1983, Noël, 2005), à la comparaison des nombres (Baroody, 1987; Van Nieuwenhoven, 1999), à l'acquisition du système arabe (Piaget, 1952), à la conservation du nombre (Piaget, 1952; Van Nieuwenhoven, 1999) et à la résolution de problèmes (Carpenter et al., 1981; Carpenter et Moser, 1984; Fayol, 1991 ; Riley et al., 1983). Dans le cadre de cette recherche, les observations ont porté plus spécifiquement sur l'utilisation des savoirs liés au comptage tel que définie par Gelman et Gallistel (1978), au dénombrement (Noël, 2005) ainsi qu'à l'abstraction qui consiste à dénombrer un ensemble hétérogène d'objets en faisant fi de certaines caractéristiques (Sophian, 1998) (ex. : associer la grosseur d'un objet à sa valeur).

\section{Les mesures de soutien de l'adulte}

Bien que l'éveil de ces savoirs soit présent à l'éducation préscolaire, certains enfants ont besoin de soutien de la part de l'enseignante. Ainsi, « en vue de poursuivre les deux grands objectifs du mandat du Programme-cycle de l'éducation préscolaire, soit de favoriser le développement global de tous les enfants et de mettre en œuvre des interventions préventives pour répondre à leurs besoins, trois orientations sont privilégiées : le jeu, l'observation du cheminement de l'enfant et l'organisation de la classe » (MEQ, 2021, p.9). En ce sens, il s'agit d'offrir un soutien par le biais du jeu de l'enfant, sous forme d'étayage et de questionnement lors des périodes de jeux, qui respecte la zone proximale de développement de l'enfant (Boily et Deshaies, 2021), ainsi que l'approche développementale prônée par le programme cycle à l'éducation préscolaire (MEQ, 2021). En fait, comme le mentionnent Gingras et ses collaborateurs (2015), la maîtrise de l'étayage permet de soutenir le développement «d'habiletés qui sont juste au-delà de ce que l'enfant est capable de réaliser seul et s'observent attentivement par le biais du jeu » (p.13). Ainsi, l'étayage de l'enseignante peut donc prendre différentes formes (Bodrova et Leong, 2011), comme le fait de questionner l'enfant sans lui donner les réponses, le faire réfléchir, soulever ses erreurs de logique sans trop insister par ailleurs, lui demander comment il a procédé, laisser des traces de sa démarche afin de lui permettre de faire des liens entre ses expériences, lui prendre la main pour l'accompagner dans le dénombrement d'objets, voire même effectuer une partie de la tâche qui lui est plus difficile. Au regard de tout ce qui peut être vu concernant l'étayage et le questionnement, nous croyons que ces différentes interventions, accompagnées des quatre rôles pouvant être occupés par les enseignantes afin de soutenir le jeu de l'enfant, pourraient favoriser l'utilisation des savoirs mathématiques de ces derniers lors du jeu symbolique. 


\section{Le développement professionnel}

Rappelons les résultats de la recherche de Casavant et Nunez-Moscoso (2020) qui sousentend une lacune dans l'acquisition des connaissances liées aux mathématiques chez les enseignantes. C'est pourquoi, l'apport d'un dispositif de formation semble une avenue à considérer. En ce sens, DeLong (2004) mentionne que pour l'acquisition des savoirs, notamment les savoirs à éveiller selon notre modèle de transposition didactique (voir figure 1), la formation, la documentation et la communauté de pratiques professionnelles sont les moyens les plus efficaces pour les acquérir. Selon le même auteur, pour l'acquisition des savoirs par l'enseignante et utilisés lors du jeu symbolique, selon notre modèle de transposition didactique (voir figure 1), le mentorat et la communauté de pratiques professionnelles sont les moyens les plus efficaces pour permettre l'acquisition de ces connaissances. Ces éléments font partie du dispositif de formation mis en place dans le cadre de cette étude.

\section{Les objectifs de cette recherche}

Afin d'apporter un éclairage sur l'adaptation du modèle de transposition didactique de Chevallard (1991) à l'éducation préscolaire, deux objectifs découlent de cette recherche :

1) Identifier la posture de l'enseignante quant à son implication dans le jeu symbolique des enfants avant et après la mise en place d'un dispositif de formation ;

2) Décrire les rôles adoptés par l'enseignante dans le jeu symbolique des enfants, à la suite d'un dispositif de formation, en lien avec l'opérationnalisation d'une transposition didactique interne des savoirs éveillés vers les savoirs utilisés en mathématiques.

\section{Méthodologie}

La présente recherche se situe dans une posture de recherche-action-formation (Charlier, 2005). Elle permet de porter un regard nouveau sur le déploiement des rôles de l'enseignante quant à l'utilisation des savoirs mathématiques des enfants lors du jeu symbolique.

\section{Les critères de sélection des classes participantes}

Afin de répondre à nos objectifs de recherche, nous avons ciblé deux classes qui mettent en place l'intervention Mathis, une intervention ludique en mathématiques au préscolaire 
(Deshaies, 2017, 2020) depuis au moins trois ans. Cette contrainte permettait de nous assurer que les situations de jeu initiées par l'enseignante (situations pédagogiques ludiques) mises en place dans les classes sont déjà connues et expérimentées de la part celles-ci. Avant la mise en place du projet de recherche, huit situations pédagogiques ludiques ont permis d'éveiller les savoirs mathématiques suivants :

- trois situations sur les savoirs liés au comptage;

- trois situations sur les savoirs liés au dénombrement;

- deux situations sur les savoirs liés à l'abstraction.

Ces situations pédagogiques ludiques se sont déroulées sur une période de huit semaines à raison d'une situation par semaine.

\section{Les participants}

Cette recherche a été réalisée auprès de deux enseignantes expérimentées (15 ans d'expérience chacune) et de 33 enfants à l'éducation préscolaire. Le groupe de l'enseignante (E1) est composé de 17 enfants (6 garçons et 11 filles) et celui de l'enseignante (E2) est composé de 16 enfants (12 garçons et 4 filles). La clientèle est issue de milieux socioéconomiques défavorisés (indice 8 selon l'IMSÉ).

\section{Les méthodes et outils de collecte et d'analyse de données}

Afin de répondre à nos deux objectifs de recherche, nous avons opté pour la passation d'un questionnaire, d'une entrevue semi-dirigée et d'observations non participantes.

\section{Les questionnaires et l'entrevue semi-dirigée}

Un questionnaire a été administré aux deux enseignantes de l'étude avant le début du projet de recherche et à la fin de celui-ci. Le questionnaire comprenait quatre questions ouvertes se regroupant en deux catégories : le rôle de l'enseignante lors du jeu symbolique et le regard de l'enseignante sur l'utilisation des savoirs mathématiques lors du jeu symbolique. Chacune de ces catégories comprenait deux questions qui permettaient aux enseignantes de relater des extraits de leur quotidien pour exemplifier leurs propos. Une entrevue semidirigée fut également réalisée à la fin du projet. Celle-ci nous a permis de demander aux enseignantes d'expliquer et d'exemplifier les rôles qu'elles occupent dans les jeux des enfants. L'entrevue ciblait deux thèmes : l'apport du dispositif de formation chez les enseignantes et le rôle de l'enseignante lors du jeu symbolique. 


\section{Les observations non participantes de la chercheure}

Des observations non participantes ont été effectuées par l'une des deux chercheuses de l'équipe. Elles consistaient à observer, et à noter les différents rôles qu'occupaient les enseignantes lors du jeu symbolique sans intervenir dans les activités de celui-ci et en se tenant à l'écart. L'observation avait pour but de décrire «les comportements et les expériences des personnes comme ils se présentent dans leurs milieux naturels » (Fortin et Gagnon, 2016, p.201). Les observations ont été détaillées à l'intérieur d'un journal de bord (Gaudreau, 2011). Par ce type d'observations, les chercheuses souhaitaient mieux comprendre le rôle adopté par les enseignantes lors des périodes de jeu symbolique et la transposition didactique interne qui s'est opérationnalisée afin d'amener les enfants à utiliser les savoirs mathématiques dans leurs jeux. Plusieurs observations ont eu lieu lors des jeux symboliques dans les deux classes et ce sur une période de quatre semaines suivant les formations offertes. Celles-ci duraient entre 50 et 60 minutes. Le contexte de jeu, les comportements des enfants et les interventions des enseignantes ont été décrits de façon détaillée dans le journal de bord.

\section{La mise en place du dispositif de formation}

Lors de 1'année scolaire 2020-2021, un dispositif de formation, comprenant des rencontres sous forme de formation et de pratique ainsi qu'un système de mentorat, a été mis en place. Trois rencontres centrées sur le rôle occupé par l'enseignante lors des jeux symboliques ont eu lieu. Ces rencontres, d'une durée de deux heures, ont permis une compréhension commune des différents rôles à adopter lors du jeu symbolique et l'utilisation des savoirs mathématiques des enfants dans ce type de jeu. Ces rencontres comprenaient un volet théorique, mais également un volet pratique lors duquel les deux enseignantes pouvaient exercer les différents rôles lors de mises en situation.

Quatre rencontres de mentorat ont eu lieu avec les enseignantes. Ces rencontres ont débuté à la suite des huit semaines consacrées à la mise en place des situations pédagogiques ludiques. Lors de ces rencontres (à raison de une par semaine), la chercheuse s'impliquait dans le jeu des enfants selon le type d'intervention qu'elle pouvait offrir à ce moment de du projet (voir tableau 1). Ces rencontres, d'une durée d'une heure, ont permis aux enseignantes d'observer la modélisation de la chercheuse quant au rôle à adopter lors du jeu symbolique. 
Tableau 1. Les types d'observation et les interventions réalisées, dans le temps, par les enseignantes lors des périodes de jeu symbolique.

\begin{tabular}{|c|c|c|c|}
\hline Semaine 1 & Semaine 2 & Semaine 3 & Semaine 4 \\
\hline $\begin{array}{l}\text { Présenter les } \\
\text { différents coins de } \\
\text { jeu symbolique aux } \\
\text { enfants sans ajout de } \\
\text { matériel issu des } \\
\text { situations } \\
\text { pédagogiques } \\
\text { ludiques. } \\
\text { Aucune intervention } \\
\text { directe ou indirecte. }\end{array}$ & $\begin{array}{l}\text { Mettre à la } \\
\text { disposition des } \\
\text { enfants, dans les } \\
\text { différents coins de } \\
\text { jeu symbolique, le } \\
\text { matériel utilisé lors } \\
\text { des situations } \\
\text { pédagogiques } \\
\text { ludiques. } \\
\text { Intervention } \\
\text { indirecte de } \\
\text { l'enseignante. }\end{array}$ & $\begin{array}{l}\text { Questionner et faire } \\
\text { preuve d'étayage } \\
\text { pour provoquer } \\
\text { l'utilisation du } \\
\text { matériel utilisé lors } \\
\text { des situations } \\
\text { pédagogiques } \\
\text { ludiques pour faire } \\
\text { émerger les savoirs } \\
\text { mathématiques. } \\
\text { Intervention de } \\
\text { soutien auprès de } \\
\text { l'enfant sans } \\
\text { implication dans le } \\
\text { jeu. }\end{array}$ & $\begin{array}{l}\text { Prendre un des quatre } \\
\text { rôles permettant de } \\
\text { soutenir le jeu } \\
\text { (observateur, metteur } \\
\text { en scène, cojoueur, } \\
\text { leader de jeu [Lemay } \\
\text { et al., 2017, adapté de } \\
\text { Johnston et al., } \\
\text { 2005]) lors des } \\
\text { périodes de jeu } \\
\text { symbolique. } \\
\text { Intervention de } \\
\text { soutien auprès de } \\
\text { l'enfant avec } \\
\text { implication dans le } \\
\text { jeu. }\end{array}$ \\
\hline
\end{tabular}

Dans le cadre de cette recherche, nous nous attardons à la semaine quatre de notre intervention (voir tableau 1) portant sur les différents rôles de l'enseignante qui permettent de soutenir le jeu des enfants pour favoriser l'utilisation des savoirs mathématiques. Rappelons toutefois que pour permettre l'utilisation des savoirs mathématiques des enfants, ceux-ci ont été éveillés préalablement par la mise en place de huit situations initiées par l'enseignante (situations pédagogiques ludiques).

\section{L'analyse des données}

Les analyses ont été réalisées à partir des réponses des questions à développement du questionnaire, des verbatims de l'entrevue semi-dirigée et des observations non participantes de la chercheure. Une analyse de contenu thématique déductive (Braun et Clarke, 2006) a été réalisée à partir du cadre théorique de Lemay et al. (2017), adapté de Johnston et al. (2005), qui ont identifié quatre rôles se situant sur un continuum démontrant l'implication de l'enseignante dans le jeu des enfants (observateur, metteur en scène, cojoueur et leader). Cette analyse nous a permis de faire ressortir des unités de sens, et ce, afin d'établir une catégorisation thématique en fonction des rôles identifiés par Lemay et al. (2017), adapté de Johnston et al. (2005). Une validation par triangulation des sources de données a été effectuée (Royer et al., 2005). Entre autres, lors des entrevues 
semi-dirigées, certaines questions supplémentaires ont été posées afin d'avoir une compréhension plus éclairée des réponses émises par les enseignantes lors des questions à développement du questionnaire (ex. : donnez un exemple du comment vous soutenez l'utilisation des savoirs mathématiques lors des jeux symboliques). Différents résultats en découlent.

\section{Résultats}

Les objectifs de recherche visaient à 1) identifier la posture de l'enseignante quant à son implication dans le jeu symbolique des enfants avant et après la mise en place d'un dispositif de formation; 2) décrire les rôles adoptés par l'enseignante dans le jeu symbolique des enfants, à la suite d'un dispositif de formation, en lien avec l'opérationnalisation d'une transposition didactique interne du savoir éveillé favorisant l'utilisation des savoirs mathématiques. Les analyses ont révélé que les enseignantes ont changé de posture à la suite du dispositif de formation mis en place et qu'elles ont adopté trois nouveaux rôles dans les jeux des enfants : observateur, cojoueur et leader. Dans cette section, à l'égard du premier objectif, les résultats de la recherche sont présentés en deux temps : 1) la posture de l'enseignante avant la mise en place du dispositif de formation ;2) la posture de l'enseignante après la mise en place du dispositif de formation. Puis, à l'égard du deuxième objectif, des exemples provenant des trois rôles adoptés par les enseignantes favorisant l'émergence des savoirs mathématiques sont décrits. À travers ces rôles, l'opérationnalisation de la transposition didactique du savoir éveillé au savoir utilisé lors des jeux symboliques est décrite.

\section{La posture de l'enseignante avant et après la mise en place du dispositif de formation}

Les analyses ont permis de constater que les deux enseignantes ont vécu un changement de posture concernant les rôles qu'elles occupent lors du jeu symbolique et de quelle façon l'utilisation de ces rôles permet à l'enfant d'utiliser ses savoirs mathématiques.

\section{Avant la mise en place du dispositif de formation}

Avant la mise en place du dispositif de formation, les enseignantes s'octroient deux rôles principaux, celui de spectateur et celui d'intervenant dans les conflits des enfants. La première enseignante (E1) fait état de son rôle de spectateur de la façon suivante : « il s'agit d'un moment où je regarde les élèves jouer. Je vois quel coin ils aiment. » La deuxième enseignante (E2) fait également état de son rôle de spectateur lorsqu'elle précise que « c'est 
un moment où les enfants jouent librement. Moi, je m'assure que tout est beau. » Nous remarquons par ces propos l'absence d'intervention pédagogique directe de la part de l'enseignante. En ce qui a trait au rôle de l'enseignante davantage associé à celui d'intervention dans la gestion de conflits qui se manifestaient lors de contextes de jeux symboliques des enfants, l'enseignante (E2) fait état de ce rôle puisqu'elle mentionne que : « il s'agit d'un moment où il y a gestion de conflit». Quant à l'enseignante (E1), face à ce rôle, elle précise qu'elle doit s'impliquer de la façon suivante : «je dois parfois gérer les amis qui veulent toujours occuper le rôle principal et les chicanes. » La posture des enseignantes nous renseigne également sur leur perception du jeu qu'elles considèrent être des moments de jeux libres. À cet effet, les enseignantes n'y voient pas nécessairement la nécessité de s'impliquer. Ainsi, outre les interventions liées à la gestion de conflit, les analyses démontrent que les enseignantes occupent un rôle non actif dans le jeu, soit celui de spectateur.

\section{Après la mise en place du dispositif de formation}

À la suite à la mise en place du dispositif de formation, l'analyse du questionnaire, de l'entrevue semi-dirigée et des observations non participantes ont révélé qu'à la suite des activités de formation (trois rencontre-formations et quatre séances de mentorat), les enseignantes se sont octroyées de nouveaux rôles lors du jeu symbolique. Conséquemment, la mise en place du dispositif de formation leur a permis de comprendre davantage comment intervenir auprès de l'enfant et a favorisé l'utilisation des savoirs mathématiques de ceux-ci selon l'adaptation du modèle de transposition didactique. Ainsi, les deux enseignantes ont mis de l'avant les rôles d'observateur, de cojoueur et de leader de jeu. La mise en place de ces rôles a permis aux enfants de transposer leurs savoirs mathématiques éveillées lors des situations pédagogiques ludiques dans le jeu symbolique. Sans l'apport $\mathrm{du}$ dispositif de formation, les enseignantes auraient possiblement maintenu leur posture initiale lors des jeux symboliques. Des exemples de ces rôles seront détaillés dans la partie qui suit.

\section{Le rôle de l'enseignante en tant qu'observateur : soutien à l'utilisation du dénombrement chez l'enfant}

L'extrait relaté ici s'est passé dans le centre de jeu symbolique du fleuriste. Deux enfants jouaient au fleuriste (un enfant occupait le rôle du fleuriste, l'autre le client). Quant à l'enseignante, elle occupait un rôle d'observateur. Dans ce rôle, l'enseignante (E2) ne s'implique pas directement dans le jeu. Toutefois, elle observe les enfants et émet un bref commentaire auprès de l'un d'entre eux. Ce rôle lui permet alors de valider la 
compréhension de l'enfant face à ses savoirs mathématiques. L'exemple suivant fait état du rôle d'observateur de l'enseignante (E2) au coin fleuriste :

Les enfants sont au coin fleuriste et il y a une affiche avec le prix des items à vendre. Un enfant veut payer un bouquet de fleurs et prend cinq bâtons à café pour représenter le $5 \$$ nécessaire. Un autre élève arrive et prend beaucoup de bâtons de café. L'enseignante lui dit : tu pourras t'acheter beaucoup de fleurs! Puis l'enfant se met à faire des paquets de cinq bâtons pour acheter des fleurs à 5 \$. Pour faire un paquet de cinq, il déplace les bâtons à un à la fois et distancie ses paquets.

Dans cet extrait, il appert qu'une transposition didactique interne du savoir éveillé au savoir utilisé chez l'enfant s'est opérationnalisée à travers l'intervention de l'enseignante. En fait, par son rôle d'observateur, l'enseignante émet un commentaire «tu pourras t'acheter beaucoup de fleurs » (E2) qui amène l'enfant à poser des actions liées au dénombrement et à s'inscrire dans la trajectoire suggérée par l'enseignante soit «l'achat » de fleurs, et plus encore «de beaucoup de fleurs ». L'intervention de l'enseignante est davantage une suggestion, mais celle-ci motive suffisamment l'enfant à utiliser son savoir lié au dénombrement éveillé lors de la situation pédagogique initiée par l'enseignante précédemment en classe (le nombre de fleurs que l'enfant pourra s'acheter avec ses bâtons) pour s'acheter «beaucoup de fleurs». L'enfant saisit donc cette situation de jeu pour utiliser son savoir lié au dénombrement. Il met alors en place le savoir de correspondance terme à terme avec les bâtons et les assemble au nombre de cinq (puisqu'avec cinq bâtons, l'enfant peut acheter des fleurs à cinq dollars) et voir combien de paquets de fleurs il peut acheter. Dès lors, le savoir lié au dénombrement est mis en œuvre.

Cette situation démontre l'opérationnalisation de la transposition didactique d'un savoir éveillé (correspondance terme à terme du dénombrement lors de la situation pédagogique ludique initiée par l'enseignante) à un savoir utilisé par l'enfant (dénombrer) pour poursuivre le jeu qu'il a commencé. L'enseignante par son rôle d'observateur a pu induire une pensée porteuse d'actions que l'enfant pourrait faire étant donné le nombre de bâtons qu'il possède (beaucoup). Cet extrait fait état de l'importance du rôle de l'enseignante dans le jeu des enfants, et ce, même si celle-ci est infime. En somme, par son intervention, l'enseignante suggère une piste d'action sur laquelle l'enfant peut investiguer dans le but de permettre (voire de favoriser) l'utilisation d'un savoir mathématique, celui de dénombrer, et ce, en situation de jeu réel.

Le rôle de l'enseignante en tant que cojoueur : soutien à l'utilisation du principe d'abstraction chez l'enfant 
Dans l'extrait qui suit, l'enseignante occupe un rôle de cojoueur. Elle participe directement au jeu et sert de modèle à l'enfant pour lui permettre l'utilisation de ses savoirs mathématiques de dénombrement et d'abstraction. L'exemple suivant fait état du rôle de cojoueur de l'enseignante dans le coin château et présente la transposition didactique interne qui s'est opérationnalisée.

Au coin château, une enfant arrive déguisée en papillon près de l'enseignante. L'enfant demande à l'enseignante si elles peuvent jouer ensemble. L'enseignante répond que oui. L'enfant lui dit qu'elle est la spectatrice et qu'elle, elle est la magicienne. L'enseignante s'assoit pour occuper son rôle de spectateur. L'enfant donne une carte avec neuf minuscules points dessus et elle dit à l'enseignante qu'elle fera apparaitre une carte avec le même nombre de points. L'enfant cherche dans son paquet de cartes et elle trouve une autre carte avec neuf points, mais de grosseur et de couleur différentes. L'enfant crie « abracadabra » et elle montre sa carte. Puis elle dit qu'elle a fait apparaitre deux cartes de neuf points. L'enseignante lui dit que les deux cartes ne sont pas pareilles. L'enfant lui dit que les deux cartes ont la même quantité de points, qu'elle doit compter le nombre de points et non regarder la couleur et la grosseur. L'enseignante lui demande si cela est identique pour les autres cartes de son paquet ou si cela fonctionne juste pour le neuf. Après réflexion, l'enfant dit que c'est pareil pour toutes les cartes et elle continue ses tours de magie.

Lors de cette observation, l'enseignante occupe un rôle de cojoueur et amène l'enfant à réfléchir sur l'abstraction de la représentation du nombre. Cet exemple démontre que le rôle de cojoueur et le questionnement de l'enseignante sur l'apparence des deux cartes amènent l'enfant à justifier ses propos et par le fait même, démontrer une acquisition du principe de l'abstraction. Quant à la transposition didactique du savoir éveillé au savoir utilisé chez l'enfant, celle-ci s'est opérationnalisée au moment où l'enseignante a saisi un savoir éveillé lors de la situation pédagogique ludique présentée précédemment (l'abstraction de la représentation du nombre) et par le biais de son intervention (les deux cartes ne sont pareilles) elle a suscité chez l'enfant l'utilisation d'un savoir mathématique, soit le principe d'abstraction de la représentation du nombre. En réalité, l'intervention de l'enseignante force l'enfant à faire part du fondement de son raisonnement et à donner le critère sur lequel se baser pour affirmer que les cartes sont bel et bien pareilles : « il faut compter le nombre de points et non regarder la couleur et la grosseur ». De plus, par sa deuxième intervention (demander si cela est identique pour les autres cartes de son paquet ou si cela fonctionne juste pour le neuf), l'enseignante a amené l'enfant à valider ses propos et à généraliser le principe d'abstraction à l'ensemble des nombres; en fait, peu importe l'apparence de la collection, un élément est égal à un (Sophian, 1998). En somme, cette situation démontre l'opérationnalisation de la transposition didactique d'un savoir éveillé 
(principe d'abstraction) à un savoir utilisé par l'enfant dans son jeu symbolique, par l'entremise du rôle de cojoueur de l'enseignante.

\section{Le rôle de l'enseignante en tant que leader de jeu : soutien à l'utilisation du principe de correspondance terme à terme}

Dans l'extrait qui suit, l'enseignante occupe un rôle de leader du jeu. Elle devient cojoueur et influence le scénario de jeu. Par ailleurs, elle utilise ce rôle pour provoquer l'utilisation du savoir lié au dénombrement. L'exemple suivant souligne une intervention réalisée par l'enseignante $(\mathrm{E} 1)$ au coin bac à sable :

Camille et Laure (noms fictifs) sont au bac à sable. Elles jouent à enterrer les diamants ; puisque le thème de la classe est princesses et chevaliers. L'enseignante arrive et demande aux enfants si elle peut jouer avec eux. Les deux filles l'acceptent dans leur jeu. L'enseignante s'installe au coin bac à sable et prend une pelle, puis elle creuse. Elle dit : wow ! Je viens de trouver un diamant ! Oh lala! J'en ai un deuxième. Les deux filles la regardent et continuent à jouer dans le sable. Puis l'enseignante dit : hum. Si nous étions des chevaliers chasseurs de trésors, combien pensez-vous que l'on pourrait trouver de diamants? Camille regarde l'enseignante et dit: je ne sais pas. Laure dit : beaucoup. Enseignante dit: Mais pour le savoir, devons-nous creuser? Alors, les deux filles se mettent à creuser et trouver des diamants. Pour chaque diamant trouvé, elles poursuivent le dénombrement (elles ne recommencent pas le dénombrement à partir de un, mais du nombre où elles sont rendues). Il y a 15 diamants en tout. Les deux filles sont heureuses, elles disent qu'elles ont un gros trésor. Puis, Laure va chercher des jetons et dit à Camille qu'elle va les cacher dans le sable et qu'elle devra lui dire combien il y en a.

Cet exemple fait état de la transposition didactique du savoir éveillé au savoir utilisé qui s'est opérationnalisée à travers l'intervention de l'enseignante. En proposant aux enfants de trouver le nombre de diamants cachés dans le bac à sable, l'enseignante saisit un savoir éveillé (dénombrement) et stimule les enfants à utiliser ce savoir dans leur jeu symbolique. Par cette activité, les fillettes sont amenées à utiliser le principe de comptage, plus précisément, le principe de correspondance terme à terme (Gelman et Gallistel, 1978). Les enfants démontrent en fait que chaque objet compté correspond à une et une seule marque représentative. Le rôle de leader de l'enseignante lui permet de proposer un défi suffisamment stimulant pour amener les enfants à participer à l'activité en posant la question suivante : « combien pensez-vous que l'on pourrait trouver de diamants? ». Ce rôle lui permet également d'orienter l'activité (on continue de creuser pour connaitre le nombre de diamants) pour amener les enfants à s'engager. Par son rôle de leader, 
l'enseignante permet aux fillettes de poursuivre le jeu en utilisant les jetons comme objet de substitution et ainsi, utiliser le dénombrement. Comme démontré dans l'extrait, sans l'intervention de l'enseignante, l'opérationnalisation de la transposition didactique d'un savoir éveillé à un savoir utilisé lors du jeu symbolique n'aurait possiblement pas eu lieu.

\section{Le rôle de soutien de l'enseignante par le questionnement et l'étayage}

Les analyses issues du questionnaire, des entrevues semi-dirigées et des observations non participantes, ont également révélé qu'à la suite des activités de formation et de la mise en place des différents rôles possibles de l'enseignante lors du jeu symbolique, le changement de posture s'est également opéré au niveau du soutien offert à l'enfant lors de son jeu symbolique. En fait, en occupant l'un ou l'autre des rôles décrits plus haut, les enseignantes avaient le souci de faire émerger les savoirs mathématiques éveillés lors des situations pédagogiques ludiques et ainsi, questionner les enfants en ce sens. Voici une observation réalisée par la chercheure concernant l'enseignante (E1) :

Deux enfants sont au bac à sable. L'enseignante les questionne sur ce qu'ils font. Ils disent qu'ils cachent des diamants. L'enseignante leur demande s'ils savent combien de diamants sont cachés. Les enfants répondent qu'ils ne le savent pas, mais ils se mettent à compter. L'enseignante quitte ce coin, mais les observe. Un enfant vient la rejoindre et lui dit qu'il y en a 11. L'enseignante lui dit : es-tu certain ? L'enfant n'est pas certain. Il retourne compter les diamants et revient vers l'enseignante pour lui dire qu'il y en a 13. L'enseignante lui demande comment il a fait. L'enfant lui prend la main et lui explique sa stratégie qui consiste à dénombrer et à déplacer les diamants comptés. Il lui dit qu'il les déplace pour ne pas se tromper.

Cette observation illustre bien l'apport du questionnement quant à l'utilisation des savoirs mathématiques éveillés lors de la situation pédagogique ludique. Par son premier questionnement, l'enseignante provoque l'envie de dénombrer de l'enfant. Puis, par son second questionnement, l'enseignante amène l'enfant à réfléchir sur sa stratégie et à la peaufiner. Enfin, lorsqu'elle lui demande comment il a fait, cela permet à l'enfant d'expliciter son savoir lié au dénombrement, plus spécifiquement la correspondance terme à terme. En fait, l'enfant utilise son savoir lié au dénombrement éveillé lors de la situation pédagogique ludique ; il s'agit du processus de transposition didactique interne. 


\section{Discussion}

Dans cette partie, nous aborderons deux constats qui ressortent de notre étude: 1) l'importance de la mise en place d'un dispositif de formation auprès des enseignantes dans le jeu symbolique des enfants concernant l'éveil et l'utilisation des savoirs mathématiques; et 2) la proposition d'un modèle de transposition didactique adapté au préscolaire et le lien à établir avec la mise en place d'un dispositif de formation.

\section{L'importance d'un dispositif de formation auprès des enseignantes dans le jeu symbolique des enfants}

Les différents résultats ont révélé un changement de posture et de pratique au niveau du rôle occupé lors du jeu symbolique. Concernant les rôles occupés par les enseignantes lors du jeu symbolique en salle de classe, les résultats démontrent qu'avant la mise en place du projet, dans les deux classes participantes, le jeu symbolique était davantage considéré comme un jeu libre, laissant les enfants à leur imagination et avec une intervention liée à la gestion de classe. Comme le mentionnent Point et Leclaire (2020), le rôle de l'enseignante lors du jeu libre « est de ne pas trop intervenir dans le jeu des enfants, mais de répondre à leurs demandes et, à l'occasion, d'aider à résoudre les conflits. Lors du jeu libre, l'intervenante n'est pas maître des apprentissages des enfants, mais plutôt observatrice » (p.52). Ce rôle est dégagé d'une forme de soutien à l'apprentissage auprès de l'enfant et le souci quant à l'utilisation des savoirs mathématiques des enfants est absent. À la suite de la mise en place du dispositif de formation, la perception de l'utilisation du jeu, de la part des deux enseignantes, a changé. Les enseignantes considèrent désormais qu'elles doivent jouer un rôle actif afin de d'amener les enfants à utiliser leurs savoirs mathématiques éveillés lors des situations pédagogiques ludiques dans un contexte de jeu symbolique. La mise en place du dispositif de formation a favorisé un changement de posture de la part des enseignantes et ainsi, un changement de relation enseignante-enfants comme proposé par Paun (2006). En fait, l'adoption des rôles de soutien des enseignantes (Lemay et al., 2017, adapté de Johnston et al., 2005), a permis la négociation entre le savoir éveillé et le savoir utilisé chez l'enfant. Sans la mise en place du dispositif de formation, cette négociation était absente lors des périodes de jeux symboliques. Ainsi, les trois rôles dorénavant occupés par les enseignantes (observateur, cojoueur et leader de jeu) leur ont permis de soutenir le jeu et d'accompagner les enfants dans celui-ci. Ces résultats s'inscrivent dans la même lignée que ceux de Parent et Bouchard (2020) sur les pratiques enseignantes dans un souci de soutien à l'acquisition du langage oral lors des jeux symboliques. Un des constats de la recherche de Parent et Bouchard (2020) est l'importance de la formation des enseignantes pour mettre en place des activités amorcées 
par l'enfant lors du jeu symbolique (savoir initié dans le jeu tel que conçu par Marinova, 2015).

Les résultats mis de l'avant ont permis de saisir l'importance du rôle de soutien et d'accompagnement joué par l'enseignante lors du jeu symbolique pour faire émerger l'utilisation des savoirs mathématiques. Comme le précisent Marinova et al., (2016), l'enseignante contribue au développement des savoirs mathématiques des enfants en s'insérant dans le jeu; en l'animant par un questionnement. Ainsi, la mise en place d'un dispositif de formation permet aux enseignantes de comprendre comment éveiller les savoirs des enfants par l'entremise des situations pédagogiques ludiques. Puis, ce dispositif permet également aux enseignantes de mieux comprendre le rôle qu'elles peuvent jouer comme soutien et accompagnement des enfants lors du jeu symbolique et ainsi, d'adopter une posture leur permettant d'opérer une perspective d'apprendre pour jouer (Marinova, 2014). En somme, par ce processus d'internalisation des actions à poser pour soutenir et accompagner l'utilisation des savoirs mathématiques, l'enseignante s'assure d'amener l'enfant à utiliser les notions mathématiques lors du jeu symbolique: il s'agit de la transposition didactique interne à l'éducation préscolaire telle que nous la concevons. En fait, cette transposition du savoir éveillé au savoir utilisé permet de rendre l'apprentissage signifiant pour l'enfant, notamment parce qu'il permet à l'enfant d'utiliser le savoir lors de situations authentiques (les jeux symboliques).

\section{La proposition d'un modèle de transposition didactique adapté au préscolaire : l'apport d'un dispositif de formation}

La mise en place d'un dispositif de formation s'avère une avenue prometteuse à explorer afin d'amener les enseignantes à mieux comprendre les différents rôles occupés lors du jeu symbolique, mais également comme soutien à l'utilisation des savoirs de l'enfant lors de ce type de jeu. En ce sens, comme le précise la recherche de Duval et al., (2016) sur la qualité des interactions des enseignants à l'éducation préscolaire, le développement de la dimension liée au soutien à l'apprentissage constitue celle ayant le plus faible niveau de qualité observé. En ce sens, le dispositif de formation, mis en place dans la présente recherche, a agi comme soutien auprès des enseignantes dans la mise en pratique du modèle de la transposition didactique à l'éducation préscolaire (voir figure 1). En fait, il s'agit du processus de transposition didactique interne des enseignantes qui, à l'éducation préscolaire, passe par la compréhension du savoir à éveiller pour soutenir l'émergence du savoir éveillé lors du jeu initié par l'enseignante (situation pédagogique ludique) et enfin, du savoir utilisé lors du jeu symbolique de l'enfant. Comme démontré par les différentes 
analyses, sans ce passage vers le savoir utilisé, peu d'enfants seraient enclins à recourir spontanément à leurs savoirs mathématiques. Ces résultats reprennent ceux de Fisher et ses collaborateurs (2012), qui mentionnent que le jeu en soi ne se semble pas suffisant pour favoriser l'émergence de l'utilisation des savoirs mathématiques : la guidance d'un adulte est requise. En somme, si nous reprenons notre adaptation du modèle de transposition didactique de Chevallard (1991), il est possible, par le rôle occupé par l'enseignante lors du jeu symbolique, de permettre aux enfants d'utiliser les savoirs éveillés lors des situations d'expérimentation ludiques dans leur jeu symbolique. En fait, lors du processus de transposition didactique, les enfants sont amenés à utiliser les savoirs, éveillés à partir des situations pédagogiques ludiques, dans leurs jeux symboliques. En outre, il appert que cette adaptation du modèle de transposition didactique trouve des fondements intéressants dans l'approche développementale. Précisément, parce que les critères suivants sont respectés : le jeu comme moyen d'apprentissage, le soutien de l'adulte aux apprentissages de l'enfant (ex. : par son questionnement ainsi que ses interventions ayant comme fondement certaines propositions d'actions possibles à entreprendre dans le jeu ou encore des orientations sous la forme de défis à relever dans le jeu, etc.), l'offre d'un environnement riche tiré de situations de la vie (ici la mise en place de coins de jeux symboliques), l'intérêt de l'enfant (l'enfant initiait ses jeux au sens où l'enseignante s'impliquait dans un jeu déjà amorcé par l'enfant) et le respect du rythme de ses apprentissages (il n'y avait aucun résultat attendu). Dans cette optique, cette démarche respecte l'approche développementale présente au sein du programme de l'éducation préscolaire (MEQ, 2021).

\section{Limites}

Il importe de faire état de certaines limites concernant les résultats de la présente recherche. D'abord, le petit nombre d'enseignantes (2) et d'enfants (33) participants ne nous permet pas de généraliser ces résultats à l'ensemble des enseignantes à l'éducation préscolaire 5 ans. Une seconde mise en place de ce projet de recherche, avec un plus grand nombre de classes, permettra certainement de mieux comprendre le processus d'utilisation des savoirs mathématiques lors du jeu symbolique et d'approfondir les rôles de l'enseignante en termes d'intervention favorisant l'utilisation de ces savoirs. De plus, cela pourrait nous amener sur des pistes quant aux divers contextes à offrir aux enfants pour utiliser ces savoirs. Cela nous permettra possiblement de cerner également le principe de généralisation des savoirs des enfants qui s'opère ou non lors du jeu symbolique. De plus, notre intérêt de recherche n'a pas porté sur le rôle que l'enfant occupe lors du jeu symbolique. Nous croyons qu'il aurait été pertinent d'en faire état afin de mieux comprendre le processus de transposition didactique interne, en vérifiant si ce dernier se traduit de façon similaire ou différente selon 
le fait que l'enfant occupe un rôle principal ou secondaire lors du scénario de jeu (Marinova, 2016). Une seconde recherche permettrait de comprendre davantage ce processus lié à l'utilisation des savoirs mathématiques lors des jeux symboliques.

\section{Conclusion}

Les résultats obtenus ont permis d'en apprendre davantage sur l'importance du rôle qu'occupe l'enseignante lors des périodes de jeu symbolique afin de soutenir l'émergence de l'utilisation des savoirs mathématiques chez les enfants. Ce rôle de soutien et d'accompagnement des enfants est nécessaire à l'utilisation des savoirs mathématiques lors de contexte de jeu (Marinova, 2016). De plus, la mise en pratique de ces différents rôles (observateur, cojoueur et metteur en scène) lors du jeu symbolique a permis l'utilisation des savoirs mathématiques des enfants. Cependant, d'autres recherches sont nécessaires afin de comprendre si un rôle joué par l'enseignante (observateur, metteur en scène, cojoueur ou leader de jeu) a un impact plus grand qu'un autre quant à l'utilisation des savoirs mathématiques. Une analyse détaillée des savoirs mathématiques utilisés par les enfants lors des différentes interventions réalisées lors des jeux symboliques serait possiblement éclairante et permettrait de mieux saisir l'ensemble des spécificités du processus de transposition interne des savoirs à l'éducation préscolaire. En terminant, cette recherche permet un éclairage nouveau sur l'adaptation du modèle de transposition didactique tel que vu par Chevallard (1991) à l'éducation préscolaire en mettant de l'avant l'importance du rôle occupé par l'enseignante lors des périodes de jeu symbolique pour permettre à l'enfant d'utiliser ses savoirs mathématiques.

\section{Références}

Baroody, A. J. (1987). Children's mathematical thinking. A developmental framework for preschool, primary, and special education teachers. Teachers College Press.

Bideaud, J., Lehalle, H., et Vilette, B. (2004). La conquête du nombre et ses chemins chez l'enfant. Presses universitaires du Septentrion.

Bodrova, E. et Leong, J. D. (2012). Les outils de la pensée : l'approche vygotskienne dans l'éducation de la petite enfance. Les Presses de l'Université du Québec.

Boily, M. et Deshaies, I. (2021) Des approches pédagogiques ayant comme fondement l'approche développementale et regard sur un modèle de développement et d'apprentissage à trois dimensions. Dans I. Deshaies et J.-M. Miron (dir.) Tisserands d'enfance tome 2, le développement de l'enfant de 4-5 ans (p. 111 à 163). JFD éditions. 
Braun, V. et Clarke, V. (2006). Using thematic analysis in psychology. Qualitative research in psychology, 3(2), 77-101.

Carpenter, T. P. Hiebert, J. et Moser, J. M. (1981). Problem structure and first-grade children's initial solution processes for simple addition and subtraction problems. Journal for Research in Mathematics Education, 12, 27-39. https://doi.org/10.2307/748656

Carpenter, T. P., et Moser, J. M. (1984). The acquisition of addition and subtraction concepts in grades one through three. Journal for Research in Mathematics Education, 15, 179-202. https://doi.org/10.2307/748348

Casavant, G. et Nunez-Moscoso, J. (2020). Vivre son insertion dans l'enseignement, entre éléments facilitateurs et obstacles. Apprendre et enseigner aujourd'hui, 9(2), 19-22.

Charlier, B. (2005). Parcours de recherche-action-formation. Revue des sciences de l'éducation, 31(2), 259-272. https://doi.org/10.7202/012755ar

Chevallard, Y. (1991). La transposition didactique : du savoir savant au savoir enseigné ( $2^{\mathrm{e}}$ éd.). La Pensée sauvage.

Clark, C. A. C., Pritchard, V. E. et Woodward, L. J. (2010). Preschool executive functioning abilities predict early mathematics achievement. Developmental Psychology, 46(5), 1176-1191. https://doi.org/10.1037/a0019672

Clements, D. H. et Sarama, J. (2012). Mathematics learning, assessment, and curriculum. Dans R. C. Pianta, L. Justice, S. W. Barnett et S. Sheridan (dir.), Handbook of early education (p. 217-239). Guilford.

Conseil Supérieur de l'Éducation (CSE). (2012). Mieux accueillir et éduquer les enfants d'âge préscolaire, une triple question d'accès, de qualité et de continuité des services. Avis à la ministre de l'Éducation, du Loisir et du Sport, Québec. Gouvernement du Québec. https://www.cse.gouv.qc.ca/wpcontent/uploads/2012/10/50-0477-AV-accueillir-eduquer-prescolaire.pdf

Dehaene, S. (2011). The number sense: How the mind creates mathematics (2 éd.). Oxford University Press.

DeLong, D.W. (2004). Lost Knowledge: Confronting the Threat of an Aging Workforce. Oxford University Press.

Deshaies, I. (2017). Effets d'une intervention didactique en mathématiques au préscolaire visant le développement $d u$ contrôle inhibiteur et adaptée au fonctionnement $d u$ cerveau sur l'apprentissage de préalables liés à l'arithmétique. Thèse de doctorat, Université du Québec à Trois-Rivières. http://depot-e.uqtr.ca/id/eprint/8031.

Deshaies, I. (2020). Mathis - Une intervention ludique en mathématiques au préscolaire. Éditions JFD 
Deshaies, I., Miron, J.-M. et Masson, S. (2015). Comprendre le cerveau des élèves pour mieux les préparer aux apprentissages en arithmétique dès le préscolaire. A.N.A.E., 27(134), 39-45.

Drainville, R. (2017). L'évaluation de l'émergence de l'écrit intégrée au jeu symbolique: étude de cas sur les pratiques d'enseignantes au préscolaire (Mémoire de maîtrise). Université du Québec en Abitibi-Témiscamingue. https://depositum.uqat.ca/id/eprint/717/

Duval, S., Bouchard, C., Hamel, C. et Pagé, P. (2016). La qualité des interactions observées en classe et déclarées par les enseignantes à l'éducation préscolaire. Revue $\begin{array}{llll}\text { canadienne de l'éducation, } & 39(3), & 1-27 .\end{array}$ https://www.jstor.org/stable/canajeducrevucan.39.3.04

Fayol, M. (1991). Du nombre à son utilisation : la résolution de problèmes additifs. Dans J. Bideaud, C. Meljac et J.-P. Fisher (dir.), Les chemins du nombre (p. 259-270). Les Presses Universitaires de Lille.

Fisher, P. H., Dobbs-Oates, J., Doctoroff, G. L. et Arnold, D. H. (2012). Early math interest and the development of math skills. Journal of Educational Psychology, 104(3), 673. https://doi.org/10.1037/a0027756

Fortin, M.-F. et Gagnon, J. (2016). Fondements et étapes du processus de recherche. Méthodes quantitatives et qualitatives. Chenelière éducation.

García coll, C., Duncan, G. J., Dowsett, C. J., Claessens, A., Magnuson, K., Huston, A. C., . . . Japel, C. (2007). School readiness and later achievement. Developmental Psychology, 43(6), 1428-1446. http://dx.doi.org/10.1037/0012-1649.43.6.1428

Gaudreau, L. (2011). Guide pratique pour créer et évaluer une recherche scientifique en éducation. Guérin éditeur

Gelman, R. (1972). The nature and development of early number concepts. Advances in Child Development and Behavior, 7, 115-167. https://doi.org/10.1016/S00652407(08)60441-3

Gelman, R. et Meck, E. (1983). Preschoolers' counting: Principles before skill. Cognition, 13(3), 343-359. https://doi.org/10.1016/0010-0277(83)90014-8

Gelman, R., et Gallistel, C.R. (1978). The child's understanding of number. Harvard University Press.

Johnson, J. E., Christie, J. F. et Wardle, F. (2005). Play, development, and early education. Allyn \& Bacon.

Lemay, L., Bouchard, C. et Bigras, N. (2017). Le jeu comme contexte pour soutenir le développement et les apprentissages des enfants: valorisé, mais méconnu et sousutilisé | Partie 2. https://passetemps.com/blogue/jeu-developpement-apprentissagedes-enfants-n3760 
Marinova, K. (2014). L'intervention éducative au préscolaire. Un modèle de pédagogie du jeu. Presses de l'Université du Québec.

Marinova, K. (2015). L'émergence de l'écrit dans le jeu : les apprentissages invisibles. Revue Préscolaire, 53(4), 9-13.

Marinova, K. (2016). Les jeux de règles et les apprentissages mathématiques. Dans K. Marinova et D. Biron (dir.), Mathématiques ludiques pour les enfants de 4 à 8 ans (p. 157-203). Presses de 1'Université du Québec.

Marinova, K. et Drainville, R. (2019). La pression ressentie par les enseignantes à adopter des pratiques scolarisantes pour les apprentissages du langage écrit à l'éducation préscolaire. Revue canadienne de l'éducation, 42(3), 606-634.

Marinova, K. Biron, D. et Drainville, R. (2016). Quand les mathématiques deviennent un jeu. Dans K.Marinova et D.Biron (dir.), Mathématiques ludiques pour les enfants de 4 à 8 ans (p.89-156). PUQ.

Marinova, K. et Biron, D. (2010). Le jeu symbolique, un contexte favorable aux apprentissages mathématiques des enfants au préscolaire. Revue préscolaire, 48(4), 23-25.

Ministère de l'Éducation du Québec. (2021). Programme-cycle d'éducation préscolaire. Éducation préscolaire. Québec : Gouvernement du Québec.

Ministère de l'Éducation et de l'Enseignement supérieur. (2017). Politique de la réussite éducative. Le plaisir d'apprendre, la chance de réussir. Québec : Gouvernement du Québec.

http://www.education.gouv.qc.ca/fileadmin/site_web/documents/education/jeunes/p feq/Programme-cycle-prescolaire.pdf

Ministère de l'Éducation et de l'Enseignement supérieur. (2018). Tout pour nos enfants. Pour la réussite éducative. Stratégie 0-8 ans. Québec: Gouvernement du Québec. http://www.education.gouv.qc.ca/fileadmin/site_web/documents/PSG/politiques_or ientations/Strate_gie_0-8_ans.pdf

Ministère de l'Éducation de l'Ontario. (2006). Guide de l'enseignement efficace des mathématiques, de la maternelle à la $\sigma^{e}$ année. http://www.atelier.on.ca/edu/resources/guides/GEE_math_M_6 fasc3.pd $\mathrm{f}$

Noël, M.-P. (2005). La dyscalculie : trouble du développement numérique de l'enfant. Éditions Solal.

Organisation de Coopération et de Développement économiques. (2007). Petite enfance, grands défis II. Éducation et structures d'accueil. Éditions OCDE. 
Pagani, L. S., Fitzpatrick, C., Archambault, I. et Janosz, M. (2010). School readiness and later achievement: A French Canadian replication and extension. Developmental Psychology, 46(5), 984-994. https://doi.org/10.1037/a0018881

Parent, A. S. et Bouchard, C. (2020). Pratiques enseignantes pour soutenir le langage oral des enfants selon les contextes de classe à l'éducation préscolaire 5 ans. INITIO, $8(1)$, $37-55$.

Paun, E. (2006). Transposition didactique : un processus de construction du savoir scolaire. Carrefours de l'éducation, 2(2), 3-13. https://doi.org/10.3917/cdle.022.0003

Piaget, J. (1952). Autobiography. Dans E. Boring (dir.), History of psychology in autobiography (Vol. 4). Clark University Press.

Point., M. et Leclaire, E. (2020). Le jeu au service du développement global de l'enfant d'âge préscolaire. Dans I. Deshaies et J.-M. Miron (dir.) Tisserands d'enfance, le développement de l'enfant de 4-5 ans (p. 45 à 67). JFD éditions.

Riley, M., Greeno, J. et Heller, J. (1983). Development of children's problem-solving ability in arithmetic. Dans H. P. Ginsburg (Dir.), The development of mathematical thinking (p. 153-196). Academic Press.

Rourke, B. P et Conway, J. A. (1997). Disabilities of arithmetic and mathematical reasoning: perspectives from neurology and neuropsychology. Journal of Learning Disabilities, 30(1), 34.

Royer, C., Guillemette, F. et Moreau, J. (2005). L'instrumentation dans la collecte des données. Recherches Qualitatives, (2), 97.

Sophian, C. (1998). A developmental perspective on children's counting. Dans C. Donlan (dir.), The development of mathematical thinking (p. 27-46). University College London Press.

Starkey, P. et Klein, A. (2000). Fostering parental support for children's mathematical development: An intervention with Head Start families. Early Education and Development, 11, 659-680.

Toub, T. S., Rajan, V., Golinkoff, R. et Hirsh-Pasek, K. (2016). Playful learning: A solution to the play versus learning dichotomy. Dans D. Berch \& D. Geary (dir.), Evolutionary perspectives on education and child development. New York, NY: Springer. 117-145.

Van Nieuwenhoven, C. (1999). Le comptage : vers la construction du nombre. De Boeck Université.

Vygotski, L.S. (1934). Pensée et langage. Éditions sociales.

Vygotski, L.S. (2012). Le problème de l'apprentissage et du développement intellectuel à l'âge scolaire. Dans F. Yvon et Y. Zinchenko (dir.), Vygotsky, une théorie du 
développement et de l'éducation : recueil de textes et commentaires (p.223-249). MGU. 